\title{
EL SISTEMA DE PRESTIGIO EN LAS UNIVERSIDADES Y EL ROL QUE OCUPAN LAS MUJERES EN EL MUNDO ACADÉMICO
}

Paulina Berríos*

* Universidad de Chile 



\section{EL SISTEMA DE PRESTIGIO EN LAS UNIVERSIDADES Y EL ROL QUE OCUPAN LAS MUJERES EN EL MUNDO ACADÉMICO}

\section{La vida privada de la universidad: una mirada al mundo académico desde la perspectiva de género}

En el presente artículo se reflexiona a partir de los hallazgos de una investigación en el ámbito de la educación superior y género en Chile ${ }^{1}$, y cuyo análisis aborda la vida privada de la educación superior; más específicamente: la vida cotidiana del cuerpo académico en el sistema universitario.

Los estudios sobre educación superior abordan generalmente aspectos que pertenecen al "ámbito de lo público" de las instituciones, es decir, se concentran en torno a cuestiones relativas al financiamiento y gobierno de las universidades, o en los procesos de acreditación y aseguramiento de la calidad, entre otros. La atención pocas veces se dirige a lo que se ha denominado "vida privada" de la universidad.

La universidad "vista por dentro" da cuenta de la experiencia vivida y percibida por el profesorado universitario. Se centra, también, en las normas y valores que orientan y definen el quehacer académico, y en cómo se instauran las reglas y se manejan los símbolos que jerarquizan constantemente las distintas funciones de docencia e investigación, así como también el "dominio de la gran ciencia" (Becher, 2001).

1 Los presentes datos y reflexiones son parte de la Tesis de Magíster en Estudios de Género y Cultura, mención Ciencias Sociales, de la Universidad de Chile de la autora. Para más información ver: http://catalogo.uchile.cl 
Las demandas no sólo se dirigen a quienes planifican, administran y coordinan el funcionamiento del sistema de educación superior, sino también, y de manera especial, a su cuerpo docente. Se considera que un cuerpo académico idóneo y altamente motivado - consciente de su importancia en el sistema universitario y en la sociedad, en tanto formadores de futuros profesionales, a la vez que responsables de producir conocimiento y tecnología para aportar al desarrollo del país- es crucial para la calidad de las instituciones de educación superior (Grupo Especial de Educación Superior y Sociedad, 2000).

Centrarse en el mundo privado posibilita una nueva interpretación y reflexión, focalizada en aspectos más cualitativos de la educación superior. Es importante conocer y comprender, en la voz de los/as académicos/as, cómo es la experiencia de trabajar en el sistema universitario. Al indagar sobre lo que significa ser "trabajadores/as del conocimiento", se observa un sistema de prestigio y poder que jerarquiza de manera constante el quehacer académico de hombres y mujeres.

En el entramado de relaciones simbólicas que se establece, podemos observar un detrimento de la función docente y una presencia mayoritaria de hombres en el ámbito de la investigación más destacada (Becher, 2001).

En un trabajo de 20 años, Becher indagó la cultura de las disciplinas en universidades norteamericanas y del Reino Unido desde un punto de vista fenomenológico. Allí pudo distinguir y reconocer las dinámicas que se han ido configurando y reproduciendo en la vida cotidiana de la universidad, y que pueden servirnos de modelo de análisis en tanto las universidades comparten una cultura intelectual, institucional y cultural propia del mundo académico.

Lo que busca el cuerpo académico no es el poder tras el cual va la clase política, ni la riqueza tras la que va el empresariado, 
sino la buena reputación. El esfuerzo que hace quien se dedica a la investigación es por lo que Becher denomina la necesidad de obtener el "renombre" o reconocimiento profesional. En virtud de las características propias de esta actividad, se depende extraordinariamente de la buena opinión de los demás, y esta reputación tiene, a su vez, muchas otras consecuencias positivas para quien produce nuevos conocimientos. Es así como, entre quienes se dedican a investigar se compite persistentemente para lograr el máximo prestigio, ya que la verdadera naturaleza de la actividad científica implica la necesidad de que otras personas del mismo campo reconozcan el valor del trabajo de quien investiga (Becher, 2001).

\section{La investigación como símbolo de reconocimiento en la universidad. El caso FONDECYT en Chile}

No siempre la investigación ha sido lo más valorado dentro del mundo académico. Sin embargo, desde que ha llegado a instaurarse como una tradición que da renombre a quienes la practican, las cifras demuestran claramente que no todos quienes participan en este mundo están en los lugares destacados de la investigación. Son sólo algunos los privilegiados, y un factor común, que se encuentra en los estudios sobre educación superior y género, es que quienes están en la producción de nuevos conocimientos y ocupando los cargos de mayor jerarquía son, mayoritariamente, hombres.

En Chile, la Comisión Nacional de Investigación Científica y Tecnológica (CONICYT) ${ }^{2}$ y, en especial, el concurso regular del Fondo Nacional de Desarrollo Científico y Tecnológico (FONDECYT) han llegado a constituirse en un referente de gran autoridad, cuyos concursos adjudicados/as por los/as investigadores/as no sólo dan

2 CONICYT actúa como institución coordinadora y articuladora del Sistema Nacional de Ciencia y Tecnología, promoviendo y fortaleciendo la investigación científica y tecnológica, la formación de recursos humanos, el desarrollo de áreas nuevas del conocimiento y de la innovación productiva, para lo cual administra los recursos públicos destinados a estas materias. Véase : http://www.conicyt.cl/acerca-conicyt/acerca.html 
prestigio de manera individual, sino también para la institución que los respalda. Esto, en parte, por los recursos que se obtienen; pero, también, porque un número creciente de proyectos aprobados le sirve a las universidades para acreditarse y darse a conocer en el exterior como organismos que desarrollan investigación.

FONDECYT se ha constituido en un concurso muy importante para académicos y académicas que dedican su tiempo a la generación de conocimiento científico y tecnológico. Ahora bien, del total de personas que presentan proyectos FONDECYT, sólo un cuarto son presentados por investigadoras responsables mujeres.

Como se observa en el gráfico $\mathrm{N}^{\circ} 1$, el número de proyectos concursados según género muestra una tendencia que no ha variado significativamente en el transcurso de casi una década.

Gráfico No 1

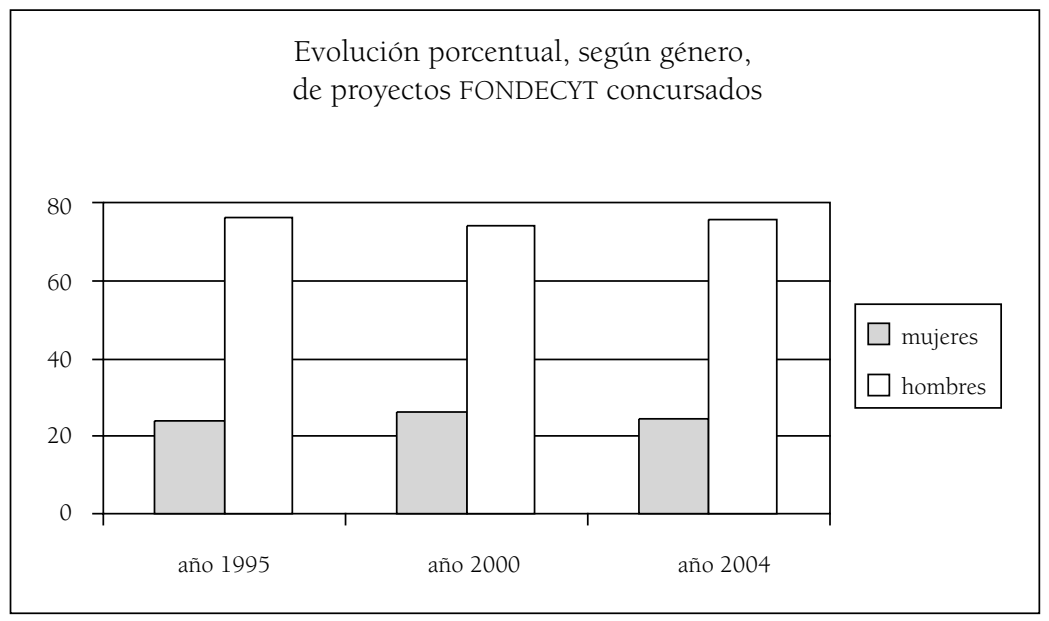

Fuente: Elaboración propia, basada en estadísticas de género de CONICYT, 2004.

A quienes han ganado concursos FONDECYT les significa ser muy bien evaluados dentro de sus respectivas instituciones. Al momento de postular a un cargo académico, ya sea para ingresar por primera vez a la universidad o para ascender de rango, los respectivos 
comités académicos (o cualquiera que sea el nombre que adquiera esta instancia de evaluación) califican los antecedentes de los/as aspirantes sobre la base de diversos indicadores, entre los que destacan, por su gravitación, el número de investigaciones y proyectos de investigación en los que participan o han participado, así como también el número de publicaciones en su trayectoria y su rendimiento productivo específico para un determinado periodo de tiempo. De acuerdo con las cifras que proporciona CONICYT, por cada proyecto aprobado que tiene una mujer como investigadora responsable, se aprueban 3,7 proyectos cuyo investigador responsable es hombre (CONICYT, 2004).

El principio de Mateo: "a los que tengan, se les dará y a los que no tengan, se les quitará incluso lo que tengan"

La presencia de las mujeres en las universidades presenta ciertas especificidades debido a las relaciones de género que se reproducen en su interior. En este contexto, las condiciones para realizar satisfactoriamente el trabajo académico no serían las mismas para las mujeres (Berríos, 2005; Guil Bozal et al., 2004; Irigoin, 1997), quienes tendrían que cumplir con estándares más altos de educación y capacitación para competir con sus colegas varones, y así neutralizar el coeficiente simbólico negativo con el que son asociadas y que afectan todo lo que son y lo que hacen (Bourdieu, 2000). En el mundo de la universidad, esto último se entrecruza con las representaciones sobre lo femenino y lo masculino, la valoración de la ciencia y su respectiva visión de mundo basada en la definición de la realidad a partir de la experimentación, la cuantificación y la técnica.

El hecho que las mujeres se sitúen en el lugar de menor proporción de proyectos aprobados en concursos de investigación como FONDECYT, trae como consecuencia que tienen menos "renombre" en la comunidad científica y que su trabajo se invisibiliza, puesto que sus nombres no se van instaurando como referentes de autoridad en sus respectivos ámbitos disciplinares, ya que no cuentan con investigaciones (con financiamiento) cuyos resultados aparezcan en publicaciones especializadas. 
Este estado de anonimato es lo que Becher (2001) señala como el "principio de Mateo": "El abismo entre la fama y el anonimato es ampliado progresivamente por la operación de lo que Merton (1973), invocando un pasaje bien conocido del primero de los cuatro evangelios, llamó el principio de Mateo: 'a los que tengan, se les dará y a los que no tengan, se les quitará incluso lo que tengan'. Mulkay (1977) se refiere a una 'estructura de elite autorreafirmante' [...] Una vez que obtenemos renombre, éste actúa como imán: logramos invitaciones para formar parte de sociedades, escribir artículos y asumir cargos".

Para las mujeres, no ganar concursos significa estar fuera de una instancia que implica no sólo prestigio sino ingresos extras. El fenómeno se complica aún más cuando se constata que no ser parte del circuito de los ganadores de concursos FONDECYT significa que tampoco se puede ser parte de los comités evaluadores.

Según los datos del gráfico $\mathrm{N}^{\circ} 2$, por cada una mujer evaluadora de proyectos presentados en los concursos regulares de FONDECYT, hay 3,4 varones en promedio (CONICYT, 2004).

Gráfico $\mathrm{N}^{\circ} 2$

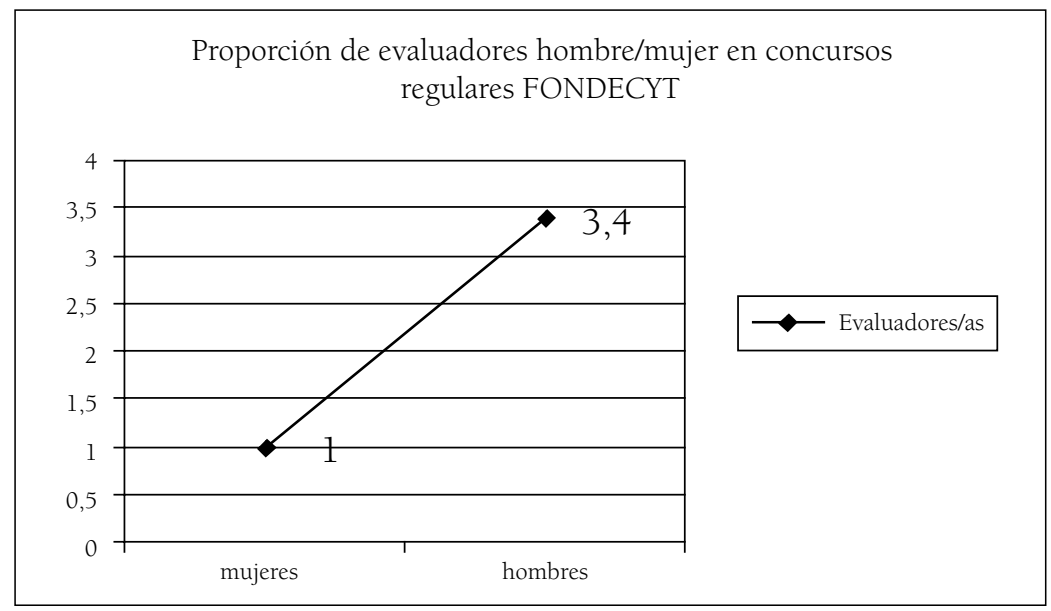

Fuente: Elaboración propia, en base a estadísticas de género CONICYT, 2004. 
Estudios cualitativos en educación superior y género han señalado que, cuando los evaluadores son en su mayoría hombres, se tiende a reproducir el imaginario de la cultura patriarcal ${ }^{3}$, que afecta de manera negativa el quehacer de las mujeres, mientras que opera una tendencia favorable y de identificación con los temas y métodos en las investigaciones realizadas por hombres. En el debate sobre educación superior y género se ha planteado lo débil que puede resultar, en el estudio de las relaciones de género, esta supuesta creencia de que los hombres, por el solo hecho de serlo, elegirían automáticamente proyectos de otros hombres, y que lo mismo ocurriría en el caso de las mujeres con otras mujeres. El conflicto por la interpretación surge de la necesidad de integrar aspectos más bien simbólicos de las relaciones de género, los cuales vienen a hacer más compleja la vida privada de la educación superior, al considerar las representaciones sociales sobre lo femenino y masculino, la dominación masculina y el paradigma científico (cartesiano) como nuevo marco referencial mental.

Cuando se observa la participación femenina en el sistema de educación superior, podemos apreciar una concentración de mujeres en carreras consideradas "feminizadas", las cuales corresponden, a su vez, a determinadas áreas y disciplinas del saber asociadas a la distinción jerárquica entre ciencias duras y ciencias blandas.

Entonces, se insinúan interrogantes acerca de las dificultades con que deben lidiar las mujeres, que van más allá de las fronteras de las universidades y que tienen que ver con el imaginario que se reproduce en el orden de la cultura patriarcal. "El poder masculino traza unas líneas de resistencia contra el acceso de las mujeres. Podríamos trazar el mapa de dichas resistencias: inferioridad numérica de las mujeres, peores puestos, tareas secundarias, etc." ${ }^{4}$

3 En este punto seguimos la definición de Castells del patriarcado, entendido como una estructura básica de todas las sociedades contemporáneas, caracterizada por la autoridad impuesta, desde las instituciones, por los hombres sobre las mujeres y sus hijos en la unidad familiar.

4 García de León, M. Antonia: Élites discriminadas (sobre el poder de las mujeres). Anthropos, Barcelona, 1994, p. 61. 
En el mundo académico, estas resistencias son construidas desde la "tribu" masculina que existe en la universidad. Podemos decir que esta tribu no es una comunidad étnica local ni un grupo indígena originario, sino un conjunto de personas del sexo masculino reunidas en torno a un ideal de comunidad de conocimiento científico, que, en la medida en que va interactuando, va generando con sus prácticas el ser y el relacionarse del mundo académico. Éste se sustenta en un principio de ordenación del mundo basado en el predominio de lo masculino, que se instituye como único referente válido para ver, sentir e interpretar la experiencia individual y de comunidad científica dentro de la universidad.

\section{Hacia políticas de equidad de género en la educación superior}

En el gráfico $\mathrm{N}^{\circ} 3$ se puede observar que, en el año 2000, el número de proyectos aprobados presentados por mujeres fue de $26,5 \%$ y por hombres de $36,8 \%$. En ese año, la diferencia fue de diez puntos porcentuales, brecha que se acorta considerablemente en 2004 cuando los porcentajes aprobados llegan casi a igualarse.

\section{Gráfico No 3}

\section{Distribución porcentual de proyectos FONDECYT aprobados según género}

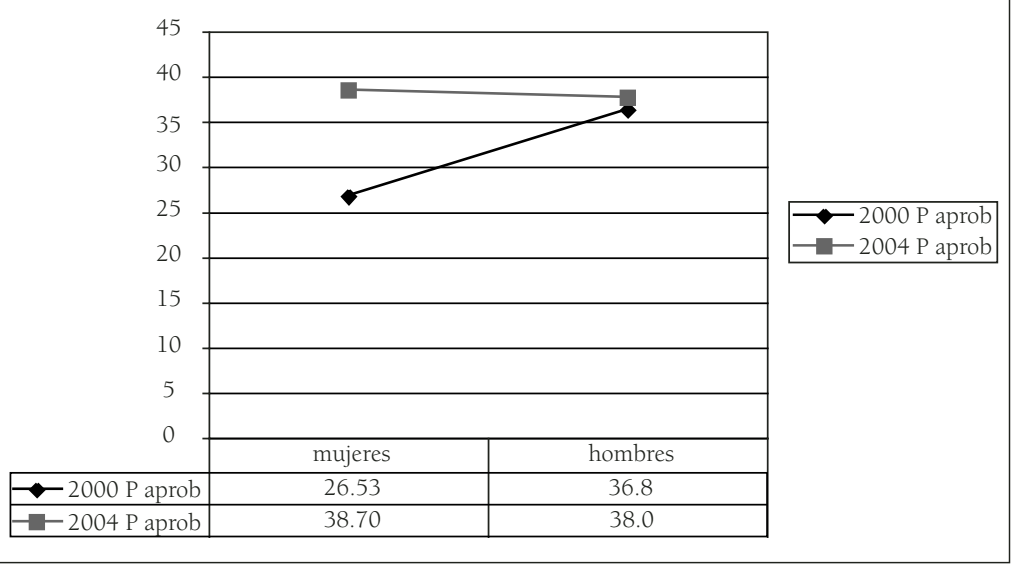

Fuente: Elaboración propia, basada en estadísticas de género CONICYT, 2004. 
¿Qué ocurre con la aprobación de proyectos con investigadoras responsables en 2004? Detenerse en este punto es interesante, pues hay una variable que podría tener incidencia al momento de conseguir una efectiva igualdad de oportunidades entre hombres y mujeres en la educación superior en Chile. Se trata de las políticas públicas de género, específicamente aquella referida al Programa de Mejoramiento de la Gestión (PMG) con enfoque de género. Este es un esfuerzo sin precedentes en América Latina, que en 2005 cumple la tercera etapa de su funcionamiento, donde la superación de las desigualdades entre hombres y mujeres deja de ser sólo el trabajo de un sector reducido o aislado, y pasa a constituirse en un mecanismo integral de coordinación estatal (Flores, 2004).

En lo relativo al sector educacional, el Servicio Nacional de la Mujer (SERNAM) ha asumido la supervisión de los PMG de género en instituciones como la Comisión Nacional de Investigación Científica y Tecnológica (CONICYT), el Consejo Superior de Educación (CSE) y la Subsecretaría de Educación, entre otros. La gestión realizada desde SERNAM, en acuerdo con CONICYT, ha significado una alianza estratégica entre instituciones estatales, que se han concertado para disminuir la diferencia de oportunidades entre quienes se mueven en el mundo de la investigación científica y tecnológica.

CONICYT, asumiendo la realidad de las cifras, se ha sensibilizado con la temática y en 2004 se apreció un claro intento por equiparar la situación entre ambos géneros. Esto llegó a reforzarse con el nombramiento, a principios de enero de 2005, de una mujer como presidenta del Consejo Superior de Ciencia FONDECYT, hecho inédito en la historia de este organismo.

Las políticas públicas de educación superior pueden crear incentivos para que el sistema universitario desarrolle una igualdad de oportunidades para hombres y mujeres del cuerpo académico. Sin embargo, son sólo las propias universidades, como instituciones, las que pueden modificar efectivamente la cultura 
de su organización, mejorar la gestión, renovar los programas de enseñanza, crear nuevos cauces de colaboración con la sociedad y volver más pertinente e internacionalmente competitiva la investigación que realizan (Brunner, 2003).

Cuando incorporamos la perspectiva de género al debate sobre educación superior, podemos plantear una serie de interrogantes y aperturas hacia futuros estudios e investigaciones. Un elemento fundamental, en el que se debe empezar a trabajar, es la confección de datos desagregados por sexo del profesorado universitario. Esta información puede ser sistematizada en una base de datos que sirva para hacer nuevos cruces de variables, que den lugar a la creación de indicadores para compensar las deficiencias estructurales que afectan a quienes desean desarrollar carrera académica en Chile. Esta dimensión cuantitativa debe complementarse, necesariamente, con estudios cualitativos sobre la educación superior.

En este contexto, cabe rescatar la estrategia de intervención planteada en la investigación "Techos de cristal en la Universidad Hispalense", pues bien podría replicarse en el contexto de las universidades chilenas. Se trata de la creación de un observatorio para la igualdad en la educación superior.

Este observatorio implicaría - entre otras - políticas públicas que incentivaran y promovieran:

- "Publicación de datos desagregados por sexo. Incluidos sobre docencia, gestión y, especialmente, investigación universitaria.

- Paridad proporcional a la presencia de las mujeres en la universidad, en todas y cada una de sus estructuras, incluidas las distintas comisiones y tribunales de evaluación.

- Premios y/o sanciones a las universidades que destaquen por su labor a favor o en contra de la igualdad de géneros.

- Lenguaje no sexista en textos y publicaciones".

5 Guil Bozal, Ana et al. Techos de Cristal en la Universidad Hispalense. Memoria de investigación. Universidad de Sevilla, noviembre 2004. 
Finalmente, podemos señalar que, hoy más que nunca, se hace necesario indagar sobre las percepciones y proyecciones que tiene el cuerpo docente sobre sus propias instituciones, descubrir el rol que cumplen y su aporte al mejoramiento de la calidad y equidad al interior del sistema de educación superior en Chile.

\section{Referencias bibliográficas}

Becher, T. Tribus y territorios académicos. La indagación intelectual y las culturas de las disciplinas. Gedisa, Barcelona, 2001.

Berríos, P. (2005) Las profesoras universitarias de género: percepciones a partir de su experiencia académica, Tesis de Grado, Magíster en Estudios de Género y Cultura, Facultad Ciencias Sociales, Universidad de Chile, 2005.

Bourdieu, Pierre. La dominación masculina. Anagrama, Barcelona, 2000.

Brunner, J. Educación superior: Chile en el contexto internacional comparado. FLACSO, Santiago de Chile, 1994.

Brunner,J. Educación superior y desarrollo en el nuevo contexto latinoamericano. Disponible en www.mecesup.cl/difusión/revista/revistalB.pdf

Castells, M. La era de la información. Volumen II El poder de la identidad. Alianza Editorial, Madrid, 1999.

Comisión Nacional de Investigación Científica y Tecnológica (CONICYT) Estadísticas de género. Documento de trabajo PMG. Santiago de Chile, octubre de 2004.

Flores, Raquel. Observaciones Informe PMG con enfoque de género. Documento interno de trabajo, SERNAM sector educación, Santiago de Chile, noviembre de 2004.

García de León, M. Antonia. Elites discriminadas (sobre el poder de las mujeres). Anthropos. Barcelona, 1994.

Grupo Especial sobre Educación Superior y Sociedad. La educación superior en los países en desarrollo. Peligros y promesas. Traducción al español, CPU, Santiago de Chile, 2000.

Guil Bozal, Ana et al. Techos de Cristal en la universidad hispalense. Memoria de investigación. Universidad de Sevilla, noviembre 2004.

Irigoin, M.E. Educación superior, equidad social y mujeres. Documento de trabajo, Santiago de Chile, julio de 1997. 